EXTENDED REPORT

\title{
Ultrasound guided synovial biopsy using portal and forceps
}

\author{
J M Koski, M Helle
}

See end of article for authors' affiliations

.....................

Correspondence to:

Dr J Koski, Department of Internal Medicine, Mikkeli Central Hospital,

Porrassalmenkatu 35-37,

Fin-50100 Mikkeli,

Finland; f.koski@fimnet.fi

Accepted

1 November 2004

Published Online First

18 November 2004
Objective: To describe a new method for taking a synovial biopsy specimen under ultrasound guidance using portal and forceps.

Methods: Percutaneous ultrasound guided biopsy was performed for 37 patients with mono- or polyarthritis as outpatients. A portal to a planned area was built using a needle, guiding wire, and dilators, through which forceps could be inserted and samples taken. Biopsy samples were taken from small and large joints, bursae, and tendon sheaths.

Results: Representative synovial tissue in adequate amounts for histopathological evaluation was obtained in $33 / 37$ cases - a success rate of $89 \%$. The biopsy procedures were well tolerated, but one complication of skin infection was encountered.

Conclusion: The new method of synovium biopsy under ultrasound guidance using sheath introducer set and flexible forceps can be performed on most joints and even bursae and tendon sheaths. The method gives sufficient samples for clinical work in most cases, but further work is needed before accepting this promising technique for scientific purposes.
$\mathrm{S}$ ynovium is an important target of arthritis research. The synovium is the primary site of inflammation and a major effector organ in a variety of joint diseases, including rheumatoid arthritis. ${ }^{2}$ Analysis of synovial tissue can provide valuable insights into the pathophysiological mechanisms associated with the aetiology, disease status, and prognosis of rheumatoid arthritis. ${ }^{3}$ In clinical work, in selected cases of unknown joint disease, it may be useful to obtain synovial membrane samples for pathological and bacteriological examinations in addition to synovial fluid analysis. ${ }^{4}$

Synovial samples can be obtained during open surgery through arthroscopy or needle arthroscopy, ${ }^{5}$ using closed needle ${ }^{6}$ or Tru-cut ${ }^{7}$ biopsy. Ultrasonography has been used for evaluating soft tissue lesions in joints, bursae, tendons, and tendon sheaths. ${ }^{9}$ In addition to diagnosing lesions, ultrasound can be used in rheumatology for interventions such as guiding injection ${ }^{10}$ and taking biopsy samples from the synovial space. ${ }^{8}$ This ability of ultrasound is based on the fact that all types of foreign bodies (instruments) are hyperechoic on sonography and can thus be detected. ${ }^{11}$ Although it has been possible to reach large as well as small joints and bursae with previous biopsy methods, ${ }^{12}{ }^{13}$ we were interested in finding an alternative outpatient method for taking synovial samples from all kinds of synovial spaces. In this article we report our experience of a new method for synovial biopsy under ultrasound guidance using an introducing set and forceps.

\section{MATERIALS AND METHODS}

A synovial biopsy was performed for 37 patients ( 16 male, 21 female) with mono- or polyarthritis. The patients' average age was 56 years (range 21-79). The diagnoses were: 17 rheumatoid arthritis, 9 unknown chronic monarthritis, 4 chronic unclassified polyarthritis, 4 seronegative spondyloarthritis, 2 psoriatic arthritis, and 1 polymyalgia rheumatica. The established diagnoses were based to the criteria of the American College of Rheumatology and the European Spondylarthropathy Study Group. ${ }^{14}{ }^{15}$ The duration of the disease was 8.5 years (range 0-33). Biopsy sites were: 23 knee, 2 elbow, 2 radiocarpal, 2 subdeltoid bursae, 2 tibiotalar, l mid-carpal, 1 glenohumeral, 1 metacarpophalangeal, 1 metatarsophalangeal joints, 1 tibialis posterior tendon, and 1 peroneus tendon sheath. The indication for the synovial biopsy was clinical: to exclude possible causes like bacterial, tuberculotic, sarcoid, pigmented villonodular, amyloid, haemochromatosis, or tumour. The same samples will also be used for later scientific purposes. Contraindications were unhealthy skin, non-cooperative patient, and anticoagulant treatment. The radiological Larsen scores ${ }^{16}$ of the biopsied joints were class L0 16, L1 14, L2 2, L3 3, L4 2, and L5 0 joints.

Percutaneous ultrasound guided biopsy was performed with the patients as outpatients. A trained nurse was needed for assistance. Premedication like sedation was not used. Esaote Technos ultrasound equipment with 5-10 and 8$13 \mathrm{MHz}$ transducers was used for localisation of effusion, synovial hypertrophy, and estimation of power Doppler signal (Esaote Biomedica, Via Siffredi 58, 16153 Genova, Italy). Disinfection of the skin was performed with Desinfektol (Berner Oy, Box 15, Fin-00131 Helsinki, Finland). The probe was covered by a sterile plastic sleeve. A sterile cloth with a hole was placed on the area involved. The operator used a mask and sterile gloves. Sterile gel was applied to the skin.

The positions of the transducer while performing biopsies were dorsal longitudinal in metacarpophalangeal, elbow, and metatarsophalangeal joints; dorsal transverse in radiocarpal and tibiotalar joints; dorsal oblique in the glenohumeral joint; anterior longitudinal in the subdeltoid bursa; and longitudinal lateral, medial, or anterior transverse in the knee joint. The probe position was longitudinal while performing the tibialis posterior or peroneus tendon sheath biopsy. These transducer positions enable the instruments to be set parallel or slightly oblique to the probe so they can be best detected with ultrasound during the procedure.

The skin and subcutaneous tissue and the synovial space involved were infiltrated with $1-10 \mathrm{ml}$ of local anaesthetic (Xylonest 1\%; Astrazeneca, Tinsdaler, Weg 183, D-22880 Wedel, Germany) under ultrasound guidance. A $4 \mathrm{~mm}$ incision of the skin with a scalpel was made and an 18 gauge needle was inserted into the planned area and 

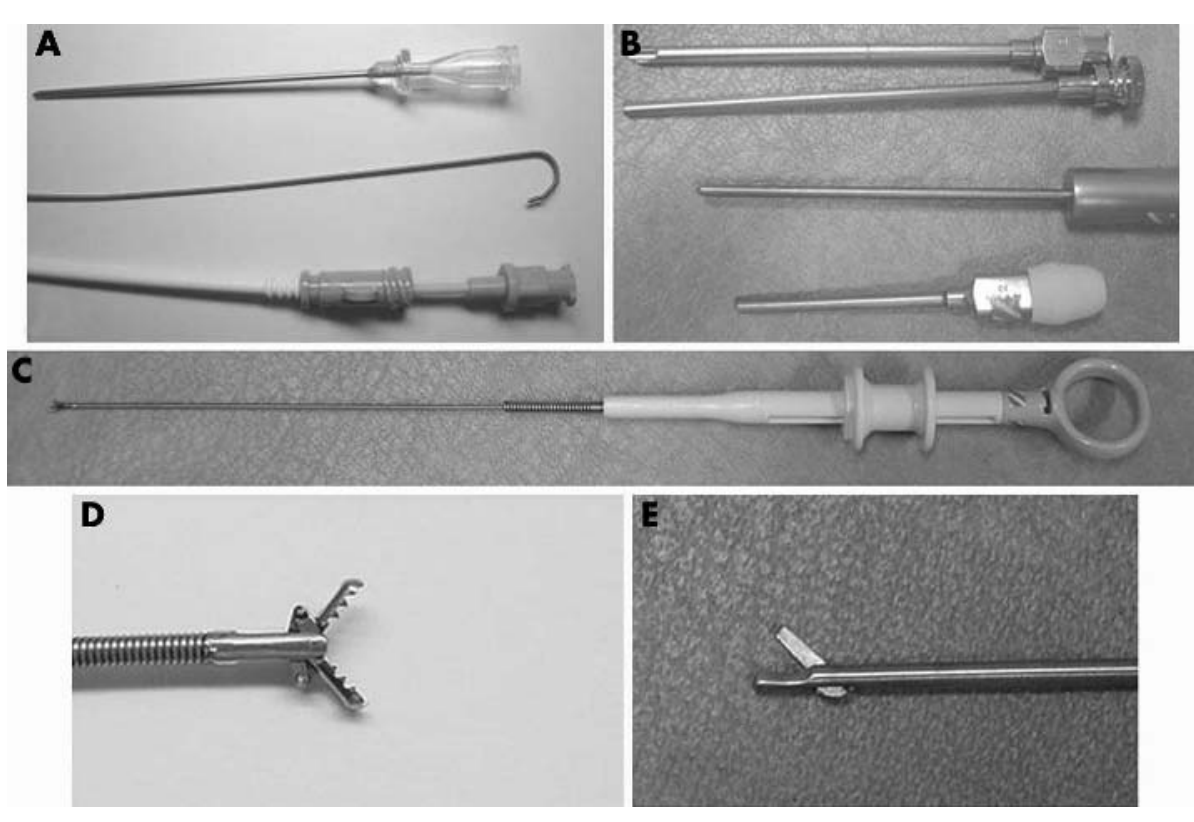

Figure 1 Synovium biopsy instruments. (A) 18 gauge needle, wire, and dilators; (B) optional metallic instruments; (C, D) Olympus FB-52C-1 forceps; and (E) rigid forceps. followed by ultrasound. The Arrow percutaneous sheath introducer set of $6 \mathrm{~F}$ contains a wire, needle, and two plastic dilators laying tightly on each other (Arrow International, Inc, 2400 Bernville Road Reading, PA 19605, USA). The wire was set into the area through the needle and this could be followed by ultrasound. The needle was removed and the two overlapping plastic or inner plastic and outer metallic dilators were set along the wire into the space. When the position was perfect, the wire and inner dilator were taken away and only the outer dilator was left and the portal was now ready for biopsy.

The excess fluid was removed. Through the portal a flexible Olympus FB-52C-1 forceps (Olympus Finland Oy, Box 134, Fin-01301 Vantaa, Finland) or a rigid biopsy forceps (Karl Storz, Mittelstrasse 8, D-78532 Tutlingen, Germany) with a diameter of $2.7 \mathrm{~mm}$ was introduced and a sample was taken.
Several samples (at least five) could be taken through the same portal, if necessary (figs 1, 2, 3, and 4). Finally, the dilator was removed and the puncture site was covered with sterile wound closure and a sterile gauze dressing. The patient was asked to keep it dry until the next day. Extra physical activity with the affected limb was forbidden for 3 days.

Biopsy specimens were immediately fixed in $4 \%$ formaldehyde for up to 24 hours, embedded in paraffin, and tissue sections were stained with standard haematoxylin and eosin. Only sections with intact synovial lining and synovial sublining were regarded as representative. Haematoxylin and eosin stained sections were scored for the extent of exudates, the hyperplasia of the lining, and the vascularity, stromal characteristics, and the inflammatory infiltrate of the sublining. The biopsy sections were assessed by the same histopathologist.
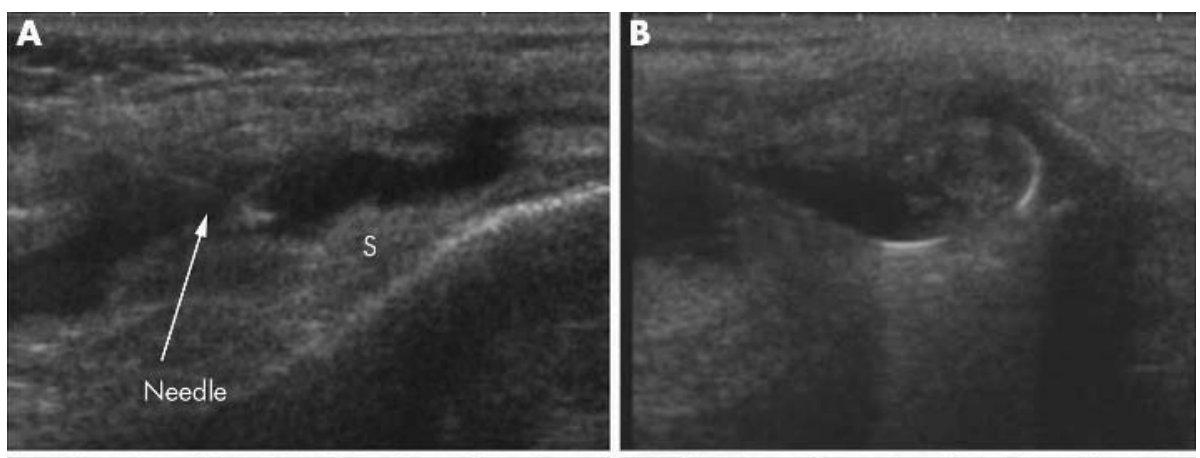

Figure 2 Synovium biopsy of the knee joint as seen with ultrasound. (A) Needle in the lateral compartment of the knee joint (longitudinal view) (S, synovium); (B) wire; (C) plastic dilator; and (D) the flexible forceps.
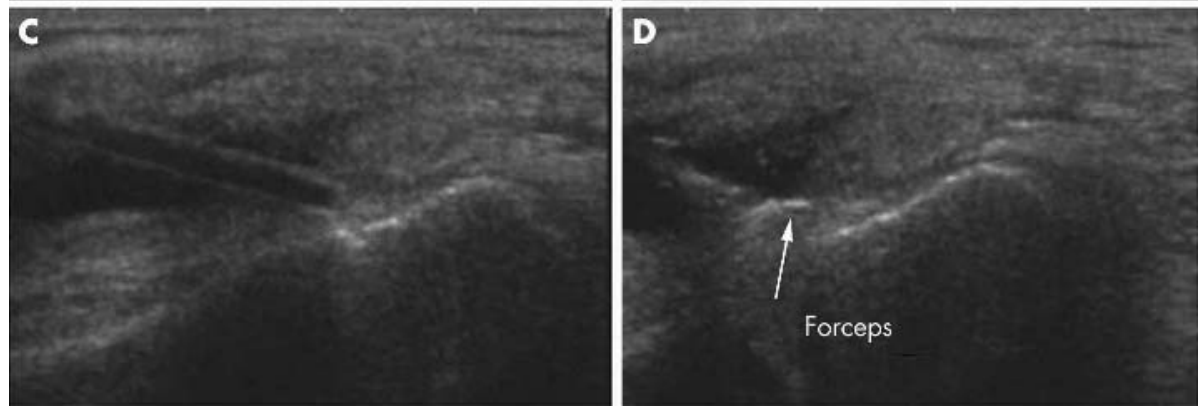

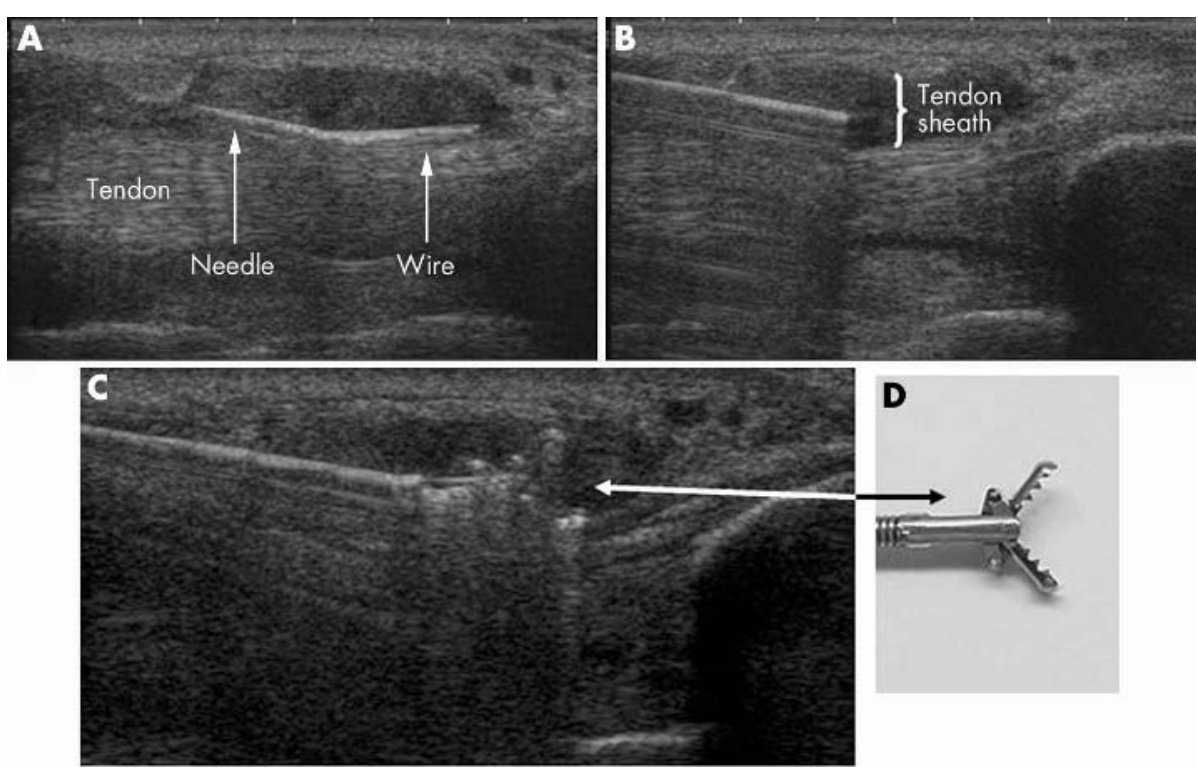

D

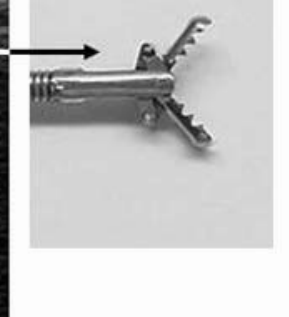

Figure 3 Synovium biopsy of the tibialis posterior tendon sheath as seen with ultrasound. (A) Needle and wire in the tendon sheath; (B) metallic dilator on the tendon; and (C, D) open jaws of the forceps ready for a bite.

\section{RESULTS}

Representative, good quality synovial tissue in adequate amount for histopathological evaluation was obtained in 33/ 37 cases-a success rate of $89 \%$. In all cases a macroscopic sample was obtained from the synovial spaces. In one knee sample examined by the pathologist the tissue contained only cartilage and fat. In another knee joint sample there was too little synovium for proper histological analysis. The third unusable sample was from the elbow joint. Here the pathologist could see a piece of joint capsule but not synovium. The fourth abortive sample was from subdeltoid bursa containing a piece of bursa wall and blood. This methodological paper is a part of a continuing trial examining the correlation of power Doppler findings with histology; these results will be published later.

Inserting the needle, local anaesthetic, and the guiding wire into the joint, bursa, or tendon sheath was an easy and rapid phase of the procedure in all cases. However, especially in large joints (knee, glenohumeral, elbow) the capsule could be thick and tough, and difficulties occurred getting the plastic dilators along the wire through the capsule. In these cases soft plastic dilators were replaced with a metal dilator

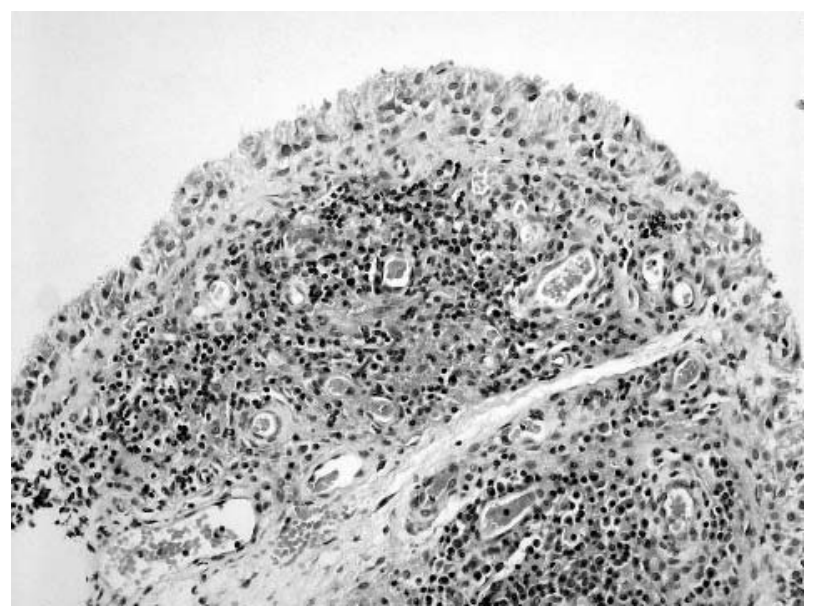

Figure 4 Histological image of the corresponding case in fig 3. The synovial lining is hyperplastic. Blood vessel proliferation and strong inflammatory infiltrate in the synovial sublining are seen. (fig 1B). Penetration of the knee joint with a large effusion was best performed with a trocar using an anterior transverse plane. In the knee joints with minimal effusion the best area for building the portal was the lateral or medial compartment using a longitudinal approach. Once the portal had been built, it was fixed and the direction could not be changed. Thus, it was important to choose the target area of the space in the diagnostic scanning phase. Because the ultrasound beam is narrow (two dimensional) the bite of the forceps could not always be seen. The flexible model winds better towards the desired target. The flexible forceps have tiny sharp teeth and the jaws turn against the synovium and thus the bite properties are better than those of the rigid model. For these reasons the flexible forceps were used in most cases and a good macroscopic sample was obtained usually with the first attempt. The metacarpophalangeal and metatarsophalangeal joints are the smallest joints for which the authors have previously performed a synovial biopsy.

The whole procedure, including local anaesthesia, disinfection, sample taking, draping, etc, took about 30-45 minutes. Synovium biopsy procedures were well tolerated. The patients did not feel pain or discomfort even during biopsy sampling, because the joint, bursa, or tendon sheath were filled with local anaesthetic. The follow up time was 6 months. However, one skin complication occurred. One week after the tibiotalar joint biopsy a 50 year old diabetic woman developed erysipelas of the same ankle. The joint aspiration culture was negative before the procedure and at the time of complication. The patient had had erysipelas of the same ankle 2 years earlier.

\section{DISCUSSION}

Synovial biopsy and analysis of synovial tissue can provide valuable insights into the pathophysiological mechanism, disease status, treatment effect, and prognosis of inflammatory joint diseases. There are two types of biopsy procedures: surgical and percutaneous. Patients requiring joint surgery are a highly selective group, and many older studies have examined synovial tissue from patients with end stage disease. Surgery involves hospital admission or day care, excessive costs, and patient discomfort (pain, scarring, and recovery). Serial biopsies are seldom possible. However, during surgery good samples can be reached under direct vision. 
Percutaneous biopsy of the synovium can be performed using needles or through arthroscopy. The most well known instrument for needle biopsy of the synovium was designed by Parker and Pearson ${ }^{6}$ and it is still used, but mostly the use is limited to the knee joint. Needle biopsy of the synovium can be performed under fluoroscopy control with the Tru-cut needle. ${ }^{7}$ Success rates have varied using different needle biopsy techniques from $48 \%$ to $95 \% .{ }^{6717}{ }^{18}$ Reasons for these differences may be technical, or the synovium is not uniformly inflamed, or the operator cannot see the synovium. When a Tru-cut biopsy of the wrist joint was performed in a small series under ultrasound guidance the success rate was $100 \%{ }^{8}$ Another office based technique developed for obtaining synovial material is the needle arthroscopy, which is regarded now as the "gold standard" for synovial biopsy sampling. The scope is $1.8-2.7 \mathrm{~mm}$ thick and samples can be selected and taken under direct vision if the two-portal system is used. ${ }^{5}$ The joints studied with this technique have been mostly knees.

Ultrasound guided synovium biopsy is somewhere between blind needle biopsy and the two-portal system needle arthroscopy. Like needle arthroscopy, ultrasound also discloses the synovial hypertrophy and vascularisation (using Doppler mode in ultrasound imaging) and the best biopsy site can thus be chosen. For both methods, after building a portal, a sample is taken at the desired site. Thus the ultrasound procedure is similar to the one-portal system of needle arthroscopy.

The relevance of the ultrasound guided biopsy method of the synovium is that it could be performed not only for the knee but also for most joints of the body, even bursae and tendon sheaths. The method worked in relatively small spaces, the metacarpophalangeal and metatarsophalangeal joints being the smallest spaces in which the biopsy was performed.

It has been a problem to reach the deeply located hip joint thus far, and we have not been able to get a sample from this joint. The introducing system was "borrowed" from angiology and the flexible forceps from gastroenterology. One manufacturer had a short model of flexible forceps suitable for rheumatological biopsies of synovial spaces. The plastic dilators used were not quite applicable for use in rheumatology, because in small joints the tools were unnecessarily long and in large joints the capsule was tough, thick, and hard to penetrate. Often a metal dilator had to be used instead of the outer plastic one. The manufacturers should develop biopsy sets for use in rheumatology: harder plastic or metallic ones with different lengths. The portal diameter of $2.7 \mathrm{~mm}$ seemed to work well as found also by the arthroscopists. ${ }^{5}$ The number of procedures performed was quite small but one complication occurred, and so, the safety of the method remains unclear. Another problem is that samples can only be taken from a selective area within the joint. Extensive research has shown that it is necessary to take samples from multiple ( six) sites within one joint to correct for sampling error, owing to the heterogeneity in the synovial tissue within one joint. ${ }^{19} 20$ This limits the implications of this technique, especially in the evaluation of treatments.

Also, although the procedure of the one-portal system of needle arthroscopy is similar to the proposed ultrasound system, it still is possible to take biopsies from multiple sites with the one-portal arthroscopy system, which is an advantage in comparison with the proposed system.

Rheumatologists who are accustomed to using musculoskeletal ultrasound and guiding needles for injection of joints and soft tissues will find it easy to perform an ultrasound guided synovium biopsy. Ultrasound guided synovium biopsy methods are an interesting alternative to other biopsy methods and they should be further developed and compared with needle arthroscopy. These methods are well tolerated techniques, which can be performed on outpatients and have low running costs. The fascinating feature of the ultrasound method using the sheath introducer set and flexible forceps is that it can be used for most joints and even for bursae and tendon sheaths. The biopsy method gives sufficient sample for clinical work in most cases, but can it provide enough representative tissue for scientific analysis? Further work is needed before accepting this promising technique for scientific purposes and the evaluation of treatments.

\section{ACKNOWLEDGEMENTS}

We are grateful to Mrs Sirkka Kosonen, manager of nursing, RN, for choosing appropriate biopsy instruments; Dr Veikko Korhonen, a gastroenterologist, for giving the excellent tip of using alligator-type forceps in joint biopsies; and nurses Anna-Maija Rautoja, Riitta Hyvärinen, Arja Vaskelainen, Minna Liimatainen, Sanna Rautio, for assistance.

\section{Authors' affiliations}

J M Koski, Department of Internal Medicine, Mikkeli Central Hospital, Porrassalmenkatu 35-37, Fin-50100 Mikkeli, Finland

M Helle, Department of Pathology, Mikkeli Central Hospital, Porrassalmenkatu 35-37, Fin-50100 Mikkeli, Finland

The study was approved by the local ethical committee and the patients gave their informed consent.

\section{REFERENCES}

1 Bresnihan B, Tak PP, Emery P, Klareskog L, Breedveld F. Synovial biopsy in arthritis research: five years of concerted European collaboration. Ann Rheum Dis 2000:59:506-10.

2 Tak PP. Analysis of synovial biopsy samples: opportunities and challenges. Ann Rheum Dis 2000;59:929-30.

3 Tak PP, Bresnihan B. The pathogenesis and prevention of joint damage in rheumatoid arthritis. Arthritis Rheum 2000;43:2619-29.

4 Schumacher HR. Synovial fluid analysis and synovial biopsy. In: Kelley WN, Harris ED, Ruddy S, Sledge CB, eds. Textbook of rheumatology. 5th ed. Philadelphia: Saunders, 1997:609-25.

5 Baeten D, van den Bosch F, Elewaut D, Stuer A, Veys EM, De Keyser F. Needle arthroscopy of the knee with synovial biopsy sampling: technical experience in 150 patients. Clin Rheumatol 1999;18:434-41.

6 Parker HR, Pearson CM. A simplified synovial biopsy needle. Arthritis Rheum 1963;6:172-6

7 Beaule V, Laredo J-D, Cywiner C, Bard M, Tubiana J-M. Synovial membrane: percutaneous biopsy. Radiology 1990;177:581-5.

8 van Vugt RM, van Dale A, Biilsma JW. Ultrasound guided synovial biopsy of the wrist. Scand J Rheumatol 1997;26:212-14.

9 Grassi W, Cervini C. Ultrasonography in rheumatology: an evolving technique. Ann Rheum Dis 1998:57:268-71.

10 Koski JM. Ultrasound guided injections in rheumatology. J Rheumatol 2000;27:2131-8

11 Horton LK, Jacobson JA, Powell A, Fessell DP, Hayes CW. Sonography and radiology of soft-tissue foreign bodies. AJR Am J Roentgenol 2001;176:1155-9.

12 Kraan MC, Reece RJ, Smeets TJ, Veale DJ, Emery P, Tak PP. Comparison of synovial tissues from the knee joints and the small joints of rheumatoid arthritis patients: implications for pathogenesis and evaluation of treatment. Arthritis Rheum 2002;46:2034-8.

13 Matsuzaki S, Yoneda M, Kobayashi Y, Fukushima S, Wakitani S. Dynamic enhanced MRI of the subacromial bursa: correlation with arthroscopic and histological findings. Skeletal Radiol 2003;32:510-20.

14 Arnett FC, Edworthy SM, Bloch DA, McShane DJ, Fries JF, Cooper NS, et al. The American Rheumatism Association 1987 revised criteria of the classification of rheumatoid arthritis. Arthritis Rheum 1988;31:315-24.

15 Dougados M, van der Linden S, Juhlin R, Huiffeldt B, Amor B, Calin A, et al. The European Spondylarthropathy Study Group preliminary criteria for the classification of spondylarthropathy. Arthritis Rheum 1991;34:1218-27.

16 Larsen A, Dale K, Eek M. Radiographic evaluation of rheumatoid arthritis and related conditions by standard reference films. Acta Radiol Diagn 1976;18:481-91.

17 Moon MS, Kim I, Kim JM. Synovial biopsy by Franklin-Silverman needle. Clin Orthop 1980; 158:224-8.

18 Schumacher HR, Kulka JP. Needle biopsy of the synovial membrane: experience with the Parker-Pearson technique. N Engl J Med 1972;286:416-19.

19 Dolhain RJ, Ter Haar NT, De Kuiper R, Nieuwenhuis IG, Zwinderman AH, Breedveld FC, et al. Distribution of T cells and signs of T-cell activation in the rheumatoid joint: implications for semiquantitative comparative histology. Br J Rheumatol 1998;37:324-30.

20 Rooney M, Condell D, Quinlan W, Daly L, Whelan A, Feighery C, et al. Analysis of the histologic variation of synovitis in rheumatoid arthritis. Arthritis Rheum 1988;31:956-63. 